\title{
Role of mtDNA-related mitoepigenetic phenomena in cancer
}

\author{
André Ferreira, Teresa L. Serafim, Vilma A. Sardão and Teresa Cunha-Oliveira \\ CNC, Center for Neuroscience and Cell Biology, University of Coimbra, Cantanhede, Portugal
}

\begin{abstract}
Background Abnormal mitochondrial function has long been associated with the development and the progression of cancer. Multiple defects in the mitochondrial genome have been reported for various cancers, however the often disregarded mitochondrial epigenetic landscape provides an additional source of deregulation that may contribute to carcinogenesis.

Design This article reviews the current understanding of mitochondrial epigenetics and how it may relate to cancer progression and development. Relevant studies were found through electronic databases (Web of Science and PubMed).

Results and conclusions The remarkably unexplored field of mitochondrial epigenetics has the potential to shed light on several cancer-related mitochondrial abnormalities. More studies using innovative, genome-wide sequencing technologies are highly warranted to assess whether and how altered mtDNA methylation patterns affect cancer initiation and progression.
\end{abstract}

Keywords Cancer, DNA methylation, mitochondria, mitoepigenetics, mtDNA.

Eur J Clin Invest 2015; 45 (S1): 44-49

\section{Introduction}

Cancer is a complex, heterogeneous disease characterized by multiple molecular and cellular transformation events that ultimately lead to the formation of tumours. A growing body of evidence suggests that defects in the collective mitochondrial genome (i.e. both nuclear and mitochondrial encoded) may be one such collection of transformation events that contribute to cancer initiation and progression. Multiple insults to the mitochondrial genome associated with cancer have been described in the literature [1,2]. For instance, characteristic mutations and deletions in control and coding regions of mitochondrial DNA (mtDNA) specific to particular cancers are also commonly reported [3], as is depletion of mtDNA [4]. In this regard, Petros et al. [5] showed that cytoplasmic hybrids (cybrids) made by fusing mitochondrial DNA depleted prostate cancer cells with mitochondria containing a well-characterized point mutation in the mtDNA-encoded ATP6 gene, known to impair ATP production and increase ROS formation, were more tumorigenic in mice than wild-type prostate cybrids. In contrast to the mitochondrial genome, little to no research efforts have been directed at the role of alterations to the mitochondrial epigenome in cancer. This review explores the potential role of the emerging field of mitoepigenetics in carcinogenesis, with particular emphasis on mtDNA epigenomics.

\section{Mitoepigenetics}

The term mitoepigenetics encompasses all bidirectional phenomena between the mitochondrial and the nuclear genomes [6]. This includes all epigenetic events that affect the expression of nuclear-encoded mitochondrial genes and, in the opposite direction, the amount of cellular mtDNA copies and the specific mtDNA haplotype - both known to significantly alter the nuclear epigenetic landscape [7]. Mitoepigenetic events may also include the interplay between mitochondrial-derived substrates and the nuclear epigenetic landscape [8,9] and the often overlooked methylation and hydroxymethylation of mtDNA, which are discussed in detail below.

\section{Mitochondrial DNA: genomics and epigenomics}

Human mtDNA is a double-stranded, closed-circular molecule of $16569 \mathrm{bp}$ that encodes for 2 rRNAs and 22 tRNAs of the mitochondrial translation machinery, as well as 13 polypeptides of the oxidative phosphorylation (OxPhos) system (Fig. 1) [10]. Similarly to nuclear DNA (nDNA), mtDNA is found in a packed protein-DNA structure called nucleoid, in which 


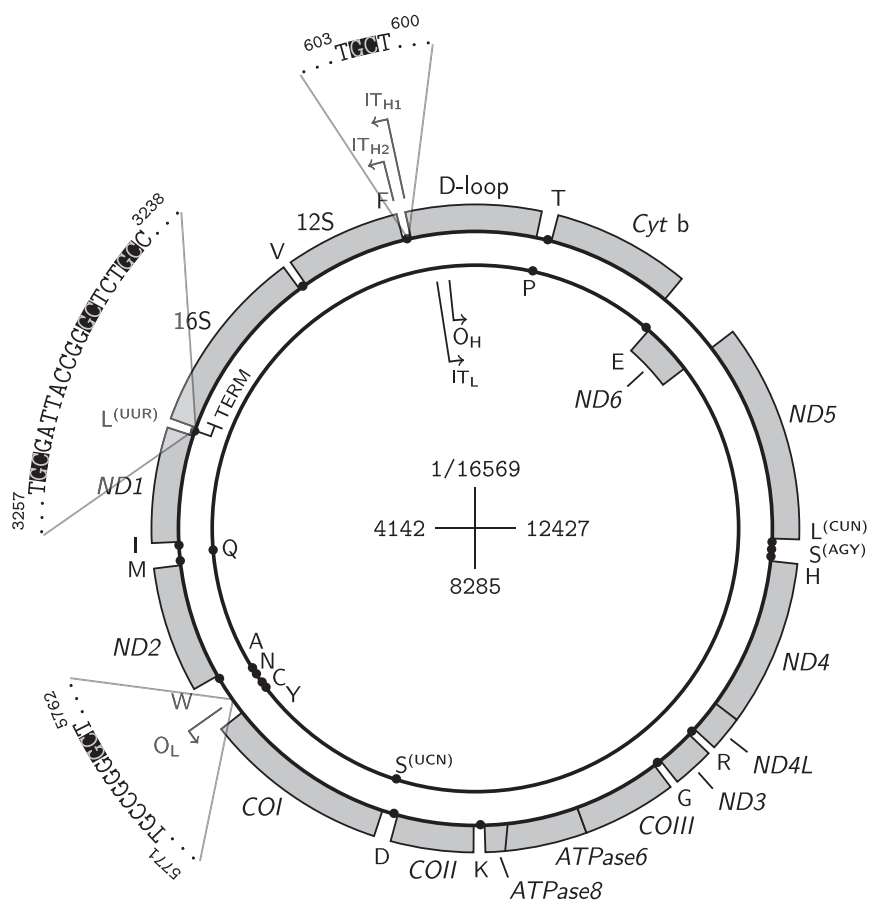

Figure 1 Map of the human mitochondrial genome, according to the revised Cambridge Reference Sequence (NC_012920, [56]). mRNA- and rRNA-coding genes (grey boxes) are interspersed with tRNA genes (black dots, single-letter code) on both the $\mathrm{H}$ - and L-strands (outer and inner circles, respectively). Duplicate tRNA genes are distinguished by their codon recognition sequences (parentheses). ND, Cyt $b, \mathrm{CO}$ and ATPase refer to genes that encode to OxPhos complexes I, III, IV and $\mathrm{V}$, respectively. Locations of the various translation initiation sites $\left(I T_{L}, I T_{H 1}, I T_{H 2}\right)$, replication origins $\left(\mathrm{O}_{H}, \mathrm{O}_{\mathrm{L}}\right)$ and the termination site (TERM), indicated by bent arrows, are in agreement with various publications (see $[10,57]$ and references therein). $\mathrm{CpGs}$ that display protection from methylation by exogenous bacterial DNMTs are highlighted in black boxes (data from [30]).

mitochondrial transcription factor A (TFAM) is the major proteic component [11]. While it is conceivable that mtDNA nucleoids may undergo epigenetic post-translational modifications in their protein scaffold in a similar fashion to histones in nDNA nucleosomes, there is currently no evidence for the occurrence of this mechanism. Thus, mtDNA epigenetic phenomena currently comprise only DNA methylation and hydroxymethylation [6].

mtDNA methylation and hydroxymethylation. The first comprehensive map of methylated cytosines $\left(\mathrm{m}^{5} \mathrm{C}\right)$ in the human mitochondrial genome was recently published [12]. Similarly to nDNA, human mtDNA displays a low frequency of CpG dinucleotides (435 in 16659 nucleotides) [13].
Although mtDNA methylation was initially reported to occur exclusively in $\mathrm{CpG}$ dinucleotides [14], recent data shows that $\mathrm{m}^{5} \mathrm{C}$ is found predominantly in non-CpG sites [15]. The origin and functional roles of non-CpG methylation are currently unknown. Some controversy regarding mtDNA methylation still remains, however, as a recent study applying regionally specific and genome-wide analyses found that both CpG methylation and hydroxymethylation were absent from human mtDNA in two different cell lines, suggesting that CpG methylation plays no role in mtDNA function [16].

Presumably, CpG mtDNA methylation is carried out by mitochondrial DNA methyltransferase (mtDNMT1), an isoform of the maintenance methylase DNMT1 that contains a mitochondrial targeting sequence [17]. The de novo methyltransferase DNMT3a may also be involved, as it has been found to colocalize with mitochondria in mouse neuronal cells when overexpressed [18]. The required methyl donor S-adenosyl-Imethionine (SAM) is synthesized in the cytosol and imported to the mitochondrial matrix via the mitochondrial SAM carrier, likely via exchange for its metabolized variant $S$-adenosyl-Ihomocysteine (SAH) [19]. SAM synthesis is regulated in part by the mitochondrial one-carbon (folate) metabolism [20]. In postmitotic differentiated cells, the mitochondrial bifunctional enzyme (MBE, a protein that participates in mitochondrial folate metabolism) is not expressed, allowing the flow of onecarbon units towards SAM synthesis. Conversely, cells with a more proliferative phenotype (e.g. embryonic or cancer cells) express MBE, and thus, one-carbon units are shuttled predominantly towards nucleotide rather than SAM synthesis $[21,22]$. Reduced SAM availability may explain, at least in part, the global nDNA hypomethylation patterns seen in cancer cells [23] and mtDNA hypomethylation in lymphoblasts derived from Down syndrome patients [24].

Levels of mtDNMT1 may also influence mtDNA methylation status. Interestingly, transcription of the mitochondrial variant of DNMT1 has been shown to be influenced by various factors related to cancer such as loss of p53 and increased signalling by the oxidative stress-responding transcription factors nuclear respiratory factor 1 (NRF1) and peroxisome proliferator-activated receptor gamma coactivator $1-\alpha$ (PGC-1 $\alpha)$ [17]. Similarly to nDNA methylation [25], mtDNA methylation can also be affected by several factors, suggesting that abnormal mtDNA methylation can be used as a biomarker [26].

Hydroxymethylation of mtDNA has also been described [17]. In nuclear DNA, $\mathrm{m}^{5} \mathrm{C}$ is oxidized into 5-hydroxymethylcytosine $\left(\mathrm{hm}^{5} \mathrm{C}\right)$ by the ten-eleven translocation (TET) family of methylcytosine dioxygenases [6]. The biological significance of this modification awaits further characterization, although it is possible that in mitochondria it promotes demethylation by preventing mtDNMT1-mediated remethylation after a replication cycle. 
mtDNA transcription. The individual strands of mtDNA are distinguished based on their different buoyant densities in denaturing $\mathrm{CsCl}$ gradients, in which they may be separated into the guanine-rich 'heavy' $(\mathrm{H})$ and cytosine-rich 'light' (L) strands. Transcription and replication of mtDNA in both strands are largely controlled by the regulatory noncoding displacement loop (D-loop) region, which contains the $\mathrm{H}$ strand promoter 1 (HSP1), the L-strand promoter (LSP) and the $\mathrm{H}$-strand origin of replication $\left(\mathrm{O}_{\mathrm{H}}\right)$ [10]. HSP2 is located close to the D-loop, roughly $\sim 60$ bp upstream of HSP1. In many types of cancer, the bulk of the reported mtDNA mutations occur predominantly in the D-loop region [3]. This indicates a trend favouring the segregation of heteroplasmic mutant D-loop mtDNA populations towards homoplasmy, as they may confer a survival advantage to cancer cells. Likewise, mtDNA variants displaying aberrant D-loop methylation could also follow this selection pattern.

Transcription of mtDNA is initiated at specific sites within the promoters $\left(\mathrm{IT}_{\mathrm{L}}, \mathrm{IT}_{\mathrm{H} 1}\right.$ and $\left.\mathrm{IT}_{\mathrm{H} 2}\right)$ and is carried out by the mitochondrial DNA-directed RNA polymerase (POLRMT), which requires the assembly of a complex with TFAM and mitochondrial transcription factor B2 (TFB2M) [27] at the promoter sites. The initial mitochondrial transcripts are polycistronic premRNAs that must be further processed to produce the individual mRNA, tRNA and rRNA molecules [28]. HSP1 produces a relatively short pre-mRNA transcript that terminates at the $3^{\prime}$ end of the 16S rRNA gene in the termination site (TERM) region, generating two tRNAs and the two rRNAs [29]. CpGs in the TERM region are strongly protected against methylation, presumably due to occupancy by the mitochondrial transcription termination factor (mTERF) that arrests HSP1-derived transcript progression at the TERM site [30]. mTERF also appears to stimulate serial HSP1 transcription [31], possibly via simultaneous binding to both the TERM and $\mathrm{IT}_{\mathrm{H} 1}$ regions (Fig. 2) [32]. Because HSP1 encodes for rRNA and tRNA only, mTERF stimulated transcription may underlie the higher rate of rRNA synthesis relative to mRNA in mitochondria [33]. HSP2 produces a much longer transcript that encompasses the majority of the H-strand, which is ultimately processed into 10 mRNAs, 13 tRNAs and the 2 rRNAs. LSP produces a transcript that spans all coding regions of the L-strand, generating $1 \mathrm{mRNA}$ and 8 tRNAs. Termination of L-strand transcription is also mediated by mTERF at the TERM site [31]. The organization of mtDNA promoters is different from that of their nuclear counterparts; nDNA promoters are enriched with clusters of $\mathrm{CpG}$ dinucleotides (the so-called 'CpG islands') [34], whereas mtDNA promoters are virtually devoid of CpG dinucleotides. While this suggests that mtDNA methylation plays no significant role in mtDNA transcription regulation, the observation that mtDNMT1 overexpression asymmetrically alters transcription rates of both mtDNA strands suggests

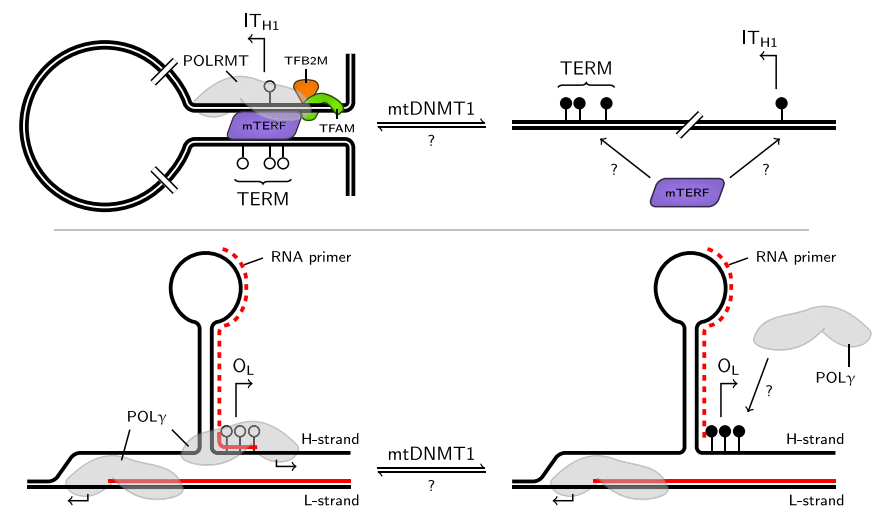

Figure 2 Schematic representation of mtDNA regulatory hubs containing $\mathrm{CpG}$ sites protected from methylation. Top: methylation of the TERM and $\mathrm{IT}_{\mathrm{H}_{1}}$ regions may alter the binding efficiency of mTERF, thereby altering normal rates of rRNA: mRNA synthesis and ultimately compromising mitochondrial translation dynamics. Bottom: methylation of $\mathrm{O}_{\mathrm{L}}$ may prevent POL $\gamma$ binding and impair mtDNA replication. Black and red strands represent parental and nascent strands, respectively. Open and solid lollipops represent unmethylated and methylated $\mathrm{CpGs}$, respectively. Presently, no data are available linking mitochondrial transcription and replication dynamics to regional mtDNA methylation status; therefore, this model should be regarded as purely hypothetical.

otherwise [17]. Moreover, some of the few CpGs that do occur in mitochondrial promoters have been found to be moderately protected from methylation by TFAM occupancy [30]. This raises the possibility that short-term reductions in TFAM levels may lead to long-term epigenetic imprinting in the transiently exposed promoters, thereby disturbing mitochondrial transcriptomics.

mtDNA replication. Replication of mtDNA occurs continuously and is independent of the cell cycle. mtDNA is replicated by a protein complex composed of polymerase $\gamma($ POL $\gamma)$, an accessory $55 \mathrm{kDa}$ subunit (p55), the replication factors mitochondrial single-stranded binding protein (mtSSB) and Twinkle, the mtDNA helicase [35]. Methylation of the POL $\gamma$ gene (POLG) promoter was shown to regulate mtDNA copy number. Replication of mtDNA begins in the $\mathrm{O}_{\mathrm{H}}$, upon extension of a cleaved LSP-derived RNA-DNA primer. Although the L-strand origin of replication $\left(\mathrm{O}_{\mathrm{L}}\right)$ is located well outside the D-loop, L-strand replication is only initiated when the growing daughter H-strand displaces the parental H-strand [28]. Thus, replication of both strands is dependent on the integrity of the D-loop. As such, abnormal methylation of the D-loop and $\mathrm{O}_{\mathrm{L}}$ regions could explain, at least in part, the depletion of 
mtDNA commonly observed in cancer. The displaced H-strand adopts a stem-loop structure with a CG-rich region at the base of the stem (Fig. 2) that is critical for L-strand replication, as mutations in this regions were shown to impair the transition from primer RNA to DNA synthesis [36]. The functional implication of $\mathrm{O}_{\mathrm{L}}$ methylation state remains to be addressed.

\section{Mitochondrial regulation of nDNA epigenetics in cancer}

Interestingly, retrograde crosstalk between mitochondria and nucleus appears to have a role on tumorigenesis [37]. Mitochondrial dysfunction can promote changes in the expression of nuclear genes involved in cellular signalling, metabolism, growth, differentiation and apoptosis [38]. There is increasing evidence that alterations in mitochondrial function severely influence nDNA methylation and histone methylation and acetylation, possibly via disturbing the normal cellular metabolome [39].

Epigenetic mechanisms involving protein acetylation are highly dependent on mitochondrial function, which affects acetyl coenzyme A availability. Tumour proliferation is promoted by upregulation of the mitochondrial citrate transport protein, which shuttles acetyl moieties from mitochondria to the cytoplasm $[40,41]$. Altered mitochondrial acetyl group export rates disrupt histone acetylation dynamics and may partly account for the enhanced tumorigenic phenotype.

Impaired function of succinate dehydrogenase (SDH), a component of both the mitochondrial respiratory chain and the Krebs cycle, promotes nDNA hypermethylation in multiple tumour lineages, including gastrointestinal stromal tumours, gliomas, paragangliomas and pheochromocytomas [42]. Similar effects were shown to occur in absence of another Krebs cycle enzyme, isocitrate dehydrogenase (IDH), suggesting that mutations or malfunction of these two Krebs cycle enzymes in distinct tumour types disturb epigenomic patterns [42]. In addition, the oncometabolite d-2-hydroxyglutarate (2HG), produced by mutant IDH enzymes, promotes neoplasia development by competitive inhibition of histone demethylation and $\mathrm{m}^{5} \mathrm{C}$ hydroxylation, leading to alterations in histone and DNA methylation in gliomas and leukaemias [43]. Human breast tumours and cancer cell lines with elevated $2 \mathrm{HG}$ also exhibit a hypermethylation phenotype, which in some cases is associated with MYC activation [44].

Absence of mtDNA in the prostate cancer cell line LN $\rho 0-8$ was shown to induce methyltransferase 1 expression and hypermethylation of the nDNA promoters, including the $\mathrm{CpG}$ islands of endothelin $\mathrm{B}$ receptor (EDNRB), $\mathrm{O}^{6}$-methylguanineDNA methyltransferase (MGMT) and E-cadherin (CDH-1) [45]. In addition, in $90 \%$ of prostate cancers, the GLI pathogenesis- related 1 (GSTP1) promoter is found methylated [46]. Distinct works show either increased or decreased mtDNA content in malignant tumours [47-49], which might depend on tumour status and origin. One such study focused on D-loop demethylation, which was pointed out as an early event in colorectal cancer that might affect mtDNA content, because the D-loop regulates mtDNA replication [47].

As several key subunits of the mitochondrial respiratory chain are coded by mtDNA, mutations or methylation of the mitochondrial genome may promote aberrant expression of those subunits, altering mitochondrial metabolism. Being the source of fundamental metabolites for enzymes that modify the epigenetic landscape of nuclear DNA, disruption of mitochondrial homeostasis may activate proto-oncogenes and/or inactivate tumour suppressing genes, leading to tumour development. In fact, oxidative phosphorylation, Krebs cycle, $\beta$-oxidation of fatty acids and amino acid and lipid metabolism are altered metabolic pathways observed in several human cancers, including colon, breast, lung, prostate, pancreas, liver, kidney and brain [50]. Thus, mtDNA methylation may occur spontaneously in normal conditions, and when a minimal threshold of mtDNA heteroplasmy is reached, changes in mitochondrial metabolism homeostasis may occur, inducing nuclear epigenetic landscape transformation, which may in turn lead to tumour formation and development.

There are some studies showing mtDNA methylation in distinct diseases $[17,51]$. As this is a rather new subject, reports regarding mtDNA methylation in cancer are currently scarce. One of the few studies available suggests that hypermethylation of mtDNA only occurs at very low frequency in both gastric and colorectal cancer [52]. However, only $37 \mathrm{CpG}$ sites were considered. A second study analysing both cervicovaginal cells of patients with cervix cancer and a cervix cancer cell line ( $\mathrm{SiHa}$ ) obtained similar results. Again, only three CpG sites were taken into account [53].

Nevertheless, it is well known that both metabolic reprogramming and epigenetic alterations are deeply involved in carcinogenesis [54,55], justifying and encouraging the search for alterations in mtDNA methylation in cancer cells using more sophisticated methodologies, such as mtDNA-wide bisulphite sequencing or LC-MS.

\section{Final remarks}

The considerations presented in this review support the idea that disrupted mitoepigenetics may contribute to tumorigenesis. Experiments aimed at elucidating the functional implications of mtDNA methylation and hydroxymethylation could help clarify the role of these epigenetic marks, deepening our understanding of mitochondrial biology in cancer and providing 
a basis for experimental treatments aimed at epigenetic manipulation.

At this stage, it is important to ascertain whether the mtDNA methylome is deregulated in various cancers and at different progression stages. This would immediately indicate new avenues of research. For instance, can aberrant mtDNA methylation be used as a reliable biomarker for cancer? Do heteroplasmic methylomes develop into homoplasmic methylomes? Is there a functional link between mtDNA methylation and mitochondrial dysfunction in cancer? Finally, does mtDNA methylation in fact play a role in cancer initiation and progression? The extreme simplicity of the mitochondrial genome relative to the nuclear genome should make it an excellent experimental model to address these issues.

\section{Acknowledgements}

Supported by Foundation for Science and Technology (FCT), Portugal, grants PTDC/DTP-FTO/1180/2012, PEst-C/ SAU/LA0001/2013-2014 and Personal Fellowship SFRH/BPD/ 75959/2011 to TLS, and cofunded by FEDER/Compete and National Budget, CENTRO-07-ST24-FEDER-002008.

\section{Conflict of interest}

The authors declare no conflict of interests.

\section{Address}

CNC, Center for Neuroscience and Cell Biology, University of Coimbra, UC Biotech Building (Lote 8A), Biocant Park, 3060-197 UC Biotech, Cantanhede, Portugal (A. Ferreira, T. L. Serafim, V. A. Sardão, T. Cunha-Oliveira).

\section{Correspondence to: Teresa Cunha-Oliveira, CNC, Center} for Neuroscience and Cell Biology, UC Biotech Building (Lote 8A), Biocant Park, 3060-197, UC Biotech, Cantanhede, Portugal. Tel.: +351 231249170 (ext 714); fax: +351 231249179; e-mails: mteroliv@cnc.uc.pt; teresa.oliveira@gmail.com

Received 20 September 2014; accepted 20 October 2014

\section{References}

1 Barbosa IA, Machado NG, Skildum AJ, Scott PM, Oliveira PJ. Mitochondrial remodeling in cancer metabolism and survival: potential for new therapies. Biochim Biophys Acta 2012;1826:238-54.

2 Brandon M, Baldi P, Wallace DC. Mitochondrial mutations in cancer. Oncogene 2006;25:4647-62.

3 Carew JS, Huang P. Mitochondrial defects in cancer. Mol Cancer 2002;1:9.

4 Lee HC, Yin PH, Lin JC, Wu CC, Chen CY, Wu CW, et al. Mitochondrial genome instability and mtDNA depletion in human cancers. Ann N Y Acad Sci 2005;1042:109-22.

5 Petros JA, Baumann AK, Ruiz-Pesini E, Amin MB, Sun CQ, Hall J, et al. mtDNA mutations increase tumorigenicity in prostate cancer. Proc Natl Acad Sci USA 2005;102:719-24.
6 Manev H, Dzitoyeva S. Progress in mitochondrial epigenetics. Biomol Concepts 2013;4:381-9.

7 Bellizzi D, D'Aquila P, Giordano M, Montesanto A, Passarino G. Global DNA methylation levels are modulated by mitochondrial DNA variants. Epigenomics 2012;4:17-27.

8 Wallace DC, Fan W. Energetics, epigenetics, mitochondrial genetics. Mitochondrion 2010;10:12-31.

9 Cyr AR, Domann FE. The redox basis of epigenetic modifications: from mechanisms to functional consequences. Antioxid Redox Signal 2011;15:551-89.

10 Falkenberg M, Larsson NG, Gustafsson CM. DNA replication and transcription in mammalian mitochondria. Annu Rev Biochem 2007;76:679-99.

11 Kanki T, Nakayama H, Sasaki N, Takio K, Alam TI, Hamasaki N, et al. Mitochondrial nucleoid and transcription factor A. Ann N Y Acad Sci 2004;1011:61-8.

12 Ghosh S, Sengupta S, Scaria V. Comparative analysis of human mitochondrial methylomes shows distinct patterns of epigenetic regulation in mitochondria. Mitochondrion 2014 DOI :10.1016/j.mito. 2014.07.007

13 Cardon LR, Burge C, Clayton DA, Karlin S. Pervasive CpG suppression in animal mitochondrial genomes. Proc Natl Acad Sci USA 1994;91:3799-803.

14 Pollack Y, Kasir J, Shemer R, Metzger S, Szyf M. Methylation pattern of mouse mitochondrial DNA. Nucleic Acids Res 1984;12:4811-24.

15 Bellizzi D, D'Aquila P, Scafone T, Giordano M, Riso V, Riccio A, et al. The control region of mitochondrial DNA shows an unusual CpG and non-CpG methylation pattern. DNA Res 2013;20:537-47.

16 Hong EE, Okitsu CY, Smith AD, Hsieh CL. Regionally specific and genome-wide analyses conclusively demonstrate the absence of CpG methylation in human mitochondrial DNA. Mol Cell Biol 2013;33:2683-90.

17 Shock LS, Thakkar PV, Peterson EJ, Moran RG, Taylor SM. DNA methyltransferase 1, cytosine methylation, and cytosine hydroxymethylation in mammalian mitochondria. Proc Natl Acad Sci USA 2011;108:3630-5.

18 Chestnut BA, Chang Q, Price A, Lesuisse C, Wong M, Martin LJ. Epigenetic regulation of motor neuron cell death through DNA methylation. J Neurosci 2011;31:16619-36.

19 Agrimi G, Di Noia MA, Marobbio CM, Fiermonte G, Lasorsa FM, Palmieri F. Identification of the human mitochondrial Sadenosylmethionine transporter: bacterial expression, reconstitution, functional characterization and tissue distribution. Biochem J 2004;379:183-90.

20 Depeint F, Bruce WR, Shangari N, Mehta R, O'Brien PJ. Mitochondrial function and toxicity: role of $\mathrm{B}$ vitamins on the onecarbon transfer pathways. Chem Biol Interact 2006;163:113-32.

21 Naviaux RK. Mitochondrial control of epigenetics. Cancer Biol Ther 2008;7:1191-3.

22 Christensen KE, MacKenzie RE. Mitochondrial one-carbon metabolism is adapted to the specific needs of yeast, plants and mammals. BioEssays 2006;28:595-605.

23 Ehrlich M. DNA methylation in cancer: too much, but also too little. Oncogene 2002;21:5400-13.

24 Infantino V, Castegna A, Iacobazzi F, Spera I, Scala I, Andria G, et al. Impairment of methyl cycle affects mitochondrial methyl availability and glutathione level in Down's syndrome. Mol Genet Metab 2011;102:378-82.

25 Levenson VV. DNA methylation as a universal biomarker. Expert Rev Mol Diagn 2010;10:481-8. 
26 Iacobazzi V, Castegna A, Infantino V, Andria G. Mitochondrial DNA methylation as a next-generation biomarker and diagnostic tool. Mol Genet Metab 2013;110:25-34.

27 Litonin D, Sologub M, Shi Y, Savkina M, Anikin M, Falkenberg M, et al. Human mitochondrial transcription revisited: only TFAM and TFB2M are required for transcription of the mitochondrial genes in vitro. J Biol Chem 2010;285:18129-33.

28 Clayton DA. Replication and transcription of vertebrate mitochondrial DNA. Annu Rev Cell Biol 1991;7:453-78.

29 Montoya J, Gaines GL, Attardi G. The pattern of transcription of the human mitochondrial rRNA genes reveals two overlapping transcription units. Cell 1983;34:151-9.

30 Rebelo AP, Williams SL, Moraes CT. In vivo methylation of mtDNA reveals the dynamics of protein-mtDNA interactions. Nucleic Acids Res 2009;37:6701-15.

31 Roberti M, Polosa PL, Bruni F, Manzari C, Deceglie S, Gadaleta MN, et al. The MTERF family proteins: mitochondrial transcription regulators and beyond. Biochim Biophys Acta 2009;1787:303-11.

32 Martin M, Cho J, Cesare AJ, Griffith JD, Attardi G. Termination factor-mediated DNA loop between termination and initiation sites drives mitochondrial rRNA synthesis. Cell 2005;123:1227-40.

33 Montoya J, Christianson T, Levens D, Rabinowitz M, Attardi G. Identification of initiation sites for heavy-strand and light-strand transcription in human mitochondrial DNA. Proc Natl Acad Sci USA 1982;79:7195-9.

34 Wang Y, Leung FC. An evaluation of new criteria for CpG islands in the human genome as gene markers. Bioinformatics 2004;20:1170-7.

35 Copeland WC. Defects in mitochondrial DNA replication and human disease. Crit Rev Biochem Mol Biol 2012;47:64-74.

36 Hixson JE, Wong TW, Clayton DA. Both the conserved stem-loop and divergent 5 -flanking sequences are required for initiation at the human mitochondrial origin of light-strand DNA replication. J Biol Chem 1986;261:2384-90.

37 Guha M, Avadhani NG. Mitochondrial retrograde signaling at the crossroads of tumor bioenergetics, genetics and epigenetics. Mitochondrion 2013;13:577-91.

38 Delsite R, Kachhap S, Anbazhagan R, Gabrielson E, Singh KK. Nuclear genes involved in mitochondria-to-nucleus communication in breast cancer cells. Mol Cancer 2002;1:6.

39 Cyr AR, Hitchler MJ, Domann FE. Regulation of SOD2 in cancer by histone modifications and $\mathrm{CpG}$ methylation: closing the loop between redox biology and epigenetics. Antioxid Redox Signal 2013;18:1946-55.

40 Catalina-Rodriguez O, Kolukula VK, Tomita Y, Preet A, Palmieri F, Wellstein A, et al. The mitochondrial citrate transporter, CIC, is essential for mitochondrial homeostasis. Oncotarget 2012;3:1220-35.

41 Kolukula VK, Sahu G, Wellstein A, Rodriguez OC, Preet A, Iacobazzi $\mathrm{V}$, et al. SLC25A1, or CIC, is a novel transcriptional target of mutant p53 and a negative tumor prognostic marker. Oncotarget 2014;5:1212-25.
42 Killian JK, Kim SY, Miettinen M, Smith C, Merino M, Tsokos M, et al. Succinate dehydrogenase mutation underlies global epigenomic divergence in gastrointestinal stromal tumor. Cancer Discov 2013;3:648-57.

43 Smolkova K, Jezek P. The role of mitochondrial NADPH-dependent isocitrate dehydrogenase in cancer cells. Int J Cell Biol 2012;2012:273947.

44 Terunuma A, Putluri N, Mishra P, Mathe EA, Dorsey TH, Yi M, et al. MYC-driven accumulation of 2-hydroxyglutarate is associated with breast cancer prognosis. J Clin Invest 2014;124:398412.

45 Xie CH, Naito A, Mizumachi T, Evans TT, Douglas MG, Cooney CA, et al. Mitochondrial regulation of cancer associated nuclear DNA methylation. Biochem Biophys Res Commun 2007;364:656-61.

46 Murphy TM, Perry AS, Lawler M. The emergence of DNA methylation as a key modulator of aberrant cell death in prostate cancer. Endocr Relat Cancer 2008;15:11-25.

47 Wen SL, Zhang F, Feng S. Decreased copy number of mitochondrial DNA: a potential diagnostic criterion for gastric cancer. Oncol Lett 2013;6:1098-102.

48 Minocherhomji S, Tollefsbol TO, Singh KK. Mitochondrial regulation of epigenetics and its role in human diseases. Epigenetics 2012;7:326-34.

49 Shen F, Huang W, Qi JH, Yuan BF, Huang JT, Zhou X, et al. Association of 5-methylcytosine and 5-hydroxymethylcytosine with mitochondrial DNA content and clinical and biochemical parameters in hepatocellular carcinoma. PLoS ONE 2013;8:e76967.

50 Gaude E, Frezza C. Defects in mitochondrial metabolism and cancer. Cancer Metab 2014;2:10.

51 Kowluru RA, Santos JM, Mishra M. Epigenetic modifications and diabetic retinopathy. Biomed Res Int 2013;2013:635284.

52 Maekawa M, Taniguchi T, Higashi H, Sugimura H, Sugano K, Kanno T. Methylation of mitochondrial DNA is not a useful marker for cancer detection. Clin Chem 2004;50:1480-1.

53 Sun C, Reimers LL, Burk RD. Methylation of HPV16 genome CpG sites is associated with cervix precancer and cancer. Gynecol Oncol 2011;121:59-63.

54 Esteller M. Epigenetics in cancer. N Engl J Med 2008;358: 11148-59.

55 DeBerardinis RJ, Lum JJ, Hatzivassiliou G, Thompson CB. The biology of cancer: metabolic reprogramming fuels cell growth and proliferation. Cell Metab 2008;7:11-20.

56 Andrews RM, Kubacka I, Chinnery PF, Lightowlers RN, Turnbull DM, Howell N. Reanalysis and revision of the Cambridge reference sequence for human mitochondrial DNA. Nat Genet 1999;23 147.

57 Taanman JW. The mitochondrial genome: structure, transcription, translation and replication. Biochim Biophys Acta 1999;1410: $103-23$. 